\title{
Simulation based Analysis of TCP Variants over MANET Routing Protocols using NS2
}

\author{
Navreet Kaur \\ M.Tech CSE Student \\ MMU Mullana(Ambala)
}

\author{
Sandhya Umrao \\ Research Scholar,CSE Dept. \\ MMU Mullana (Ambala)
}

\author{
Rajneesh Kumar Gujral, \\ Ph.D \\ Prof. CSE Dept. \\ MMU Mullana(Ambala)
}

\begin{abstract}
Traditional Transport Layer Protocol Transport Control Protocol (TCP) and User Datagram Protocol (UDP) which perform well on wired networks, but it degrades its performance in case of Ad-hoc wireless networks due to the existing problems associated such as misinterpretation of packet loss, frequent path breaks, effect of path length, misinterpretation of congestion windows, asymmetric link behaviour, uni-directional path, multipath routing and the use of sliding-window-based transmission. So, to provide reliable data communication support over MANET's, various TCP variants i.e. basically enhancement of base TCP protocols has been proposed. This research work aims to evaluating the simulation based comparison of two TCP variants, which are TCP Vegas and TCP Westwood on different routing protocols such as AODV, DSDV and DSR on the basis of average throughput, packet delivery ratio, average delay, routing overhead and average jitter with the use of ns 2 simulator.
\end{abstract}

\section{Keywords}

TCP Vegas, TCP Westwood, MANET, NS2

\section{INTRODUCTION}

Mobile Ad-hoc Networks (MANETs) are self -configuring networks consisting of mobile nodes that are communicating through wireless links. There is a cooperative engagement of a collection of mobile nodes without the required intervention of any centralized access point or existing infrastructure. The nodes move arbitrarily; therefore, the network may experience unpredictable topology changes. It means that a formed network can be deformed on the fly due to mobility of nodes. Hence, it is said that an Ad-hoc wireless network is self organizing and adaptive. Due to infrastructure less and selforganizing nature of Ad-hoc networks, it has several applications in the area of commercial sector for emergency rescue operations and disaster relief efforts. MANETs also provides a solution in the field of military battlefield to detect movement of enemies as well as for information exchange among military headquarters and so on [1]. Also, MANET provides an enhancement to cellular based mobile network infrastructure. Nowadays, it is an inexpensive alternative for data exchange among cooperative mobile nodes [2].

\section{OVERVIEW OF ROUTING AND TRANSPORT LAYER PROTOCOLS}

\subsection{Routing Protocol}

The routing is the act of transferring information (packets) across a network from a source to a destination. The routing infrastructure needs to be established in a distributed, self- organized way due to node mobility. The MANET routing protocols can be divided into three categories [3].

- Proactive protocols or table driven protocols

- Reactive protocols or on-demand protocols

- Hybrid Routing protocols

Ad-hoc on demand distance vector (AODV): It is an on-demand and distance-vector routing protocol, meaning that a route is established by AODV from a destination only on demand [4]. AODV is capable of both unicast and multicast routing [5]. It keeps these routes as long as they are desirable by the sources. It is loop-free, self-starting, and scales to large numbers of mobile nodes [6] [7]. AODV defines three types of control messages for route maintenance i.e., RREQ, RERR and RREP. The main advantage of AODV is that it is a beacon-full routing protocol so that its convergence is fast due to linked-node communication and disadvantage of AODV is that the periodic beaconing leads to unnecessary bandwidth consumption.

Destination-Sequenced Distance Vector (DSDV): DSDV is a table-driven routing [8] scheme for MANETs. The Destination-Sequenced Distance-Vector (DSDV) Routing Algorithm is based on the idea of the classical Bellman-Ford Routing Algorithm with certain improvements. Every mobile station maintains a routing table that lists all available destinations, the number of hops to reach the destination and the sequence number assigned by the destination node. The sequence number is used to distinguish stale routes from new ones and thus avoid the formation of loops.

Dynamic Source Routing (DSR): DSR is an on-demand protocol designed to restrict the bandwidth consumed by control packets in ad hoc wireless networks by eliminating the periodic table-update messages required in the table-driven approach [9]. The major difference between this and other ondemand routing protocols is that it is beacon-less and hence does not require periodic hello packet (beacon) transmission, which are used by a node to inform its neighbours of its presence. The basic approach of this protocol (and all other on-demand routing protocols) during the route construction phase is to establish a route by flooding Route Request packets in the network. The destination node, on receiving a Route Request packet, responds by sending a Route Reply packet back to the source, which carries the route traversed by the Route Request packet received.

\subsection{TCP Protocol}

Transmission Control Protocol (TCP)[10][11] is the predominant Internet protocol and it carries approximately $90 \%$ of Internet traffic in today's heterogeneous wireless and 
wired networks. TCP is reliable end to end protocol because TCP is trying to provide reliable data transmission between two entities. TCP is widely used as a connection oriented transport layer protocol that provides reliable data packet delivery over unreliable links. TCP primary purpose is to provide a connection -oriented reliable data transfer service between different applications to be able to provide these services on top of an unreliable communication system. TCP needs to consider data transfer, reliability flow control, multiplexing, TCP segment, and congestion control and connection management. TCP does not depend on the underlying network layers and, hence, design of various TCP variants is based on the properties of wired networks.

TCP Vegas: Vegas is a TCP implementation which is a modification of RENO. It builds on the fact that proactive measure to encounter congestion is much more efficient than reactive ones. It tried to get around the problem of coarse grain timeouts by suggesting an algorithm which checks for timeouts at a very efficient schedule. Also it overcomes the problem of requiring enough duplicate acknowledgements to detect a packet loss, and it also suggests a modified slow start algorithm which prevents it from congesting the network.

TCP Westwood: TCP Westwood congestion control algorithm [12] use a bandwidth estimation, it executed at sender side of a TCP connection. The congestion window dynamics during slow start and congestion avoidance are unchanged. The general idea is to use the bandwidth estimate BWE to set the congestion window (cwin) and the slow start threshold (ssthresh) after a congestion episode. In TCP Westwood the sender continuously computes the connection BWE which is defined as the share bottleneck used by the connection. Thus, BWE is equal to the rate at which data is delivered to the TCP receiver. The estimate is based on the rate at which ACKs are received and on their payload. After a packet loss, the sender resets the congestion window and the slow start. Threshold based on BWE. The packet loss is suspected with a reception of three duplicates ACKs or timeout expiration. Another important element of this procedure is the RTT estimation. That is because the congestion window is set precisely to BWE * RTT after indication of packet loss.

\section{RELATED WORK}

Extensive literature survey has been done to find the gaps TCP Variants over MANET Routing Protocols. Yuvaraju B N et al. [13] performed the simulation based analysis of variants of TCP on the three performance metrics such as Throughput, Average End-to-End delay and Packet Delivery ratio in low and high mobility by using ns2 simulator. After analysing the performance from simulated data and graphs obtained, we concluded that out of six TCP variants named TCP TAHOE, TCP RENO, TCP NEW RENO, TCP SACK, TCP FACK and TCP Vegas, performance of TCP Vegas is better for sending data and information due to its better packet delivery ratio and avg. End-to-End delay in both high and low mobility. B.S. Yew et al. [14] performed the simulation based analysis of TCP Vegas versus different TCP variants in homogenous and heterogeneous networks by using network simulator (ns-2). After simulation, it was observed that the overall performances of TCP variants in wired-cum-wireless network are poorer compared to their performances in wired network. It was also observed that TCP Vegas always exhibits significant lower delay as compared to other TCP variants in both wired and wired-cum-wireless network. MACURA et al. [15] described the evaluation and comparison of three control algorithms, which are Westwood+, New Reno and Vegas TCP by using ns2. Results show that Westwood+ TCP is friendly towards New Reno TCP and improves the fairness in bandwidth allocation whereas Vegas TCP is fair but is not able to grab its bandwidth share when coexisting with Reno or in the presence of reverse traffic because of its RTT-based congestion detection mechanism. Final results show that Westwood+ remarkably improves utilization of wireless links that are affected by losses not due to congestion.

Rajneesh Kumar Gujral et al [16] has performed Analysis that at what speed and by taking how much zone radius ZRP will be able to perform efficiently and effectively for MANETs. Give a results that if the radius zone is small then the nodes act as reactive protocol so if the zone is less than the average delay is more. When the mobility rate is less then throughput, packet delivery ratio is maximum and if the mobility rate and zone size is increase the control overhead is also increased.

Simulation based analysis of TCP and UDP over ZRP has been analyzed in [17]. After analysis its results have concluded that, when zone size is very small it act as reactive routing protocol because the probability of destination node with in routing zone is less, so average delay is more. ZRP uses proactive routing within the zone as zone size gets increased then delay keeps on reducing destination nodes can come under the routing zone. As TCP is reliable protocol. When it has been analyzed on ZRP, its results shows maximum packet delivery ratio with lower mobility speed and lowest packet delivery ratio with highest mobility speed. Throughput is also inversely proportionate to mobility speed and zone size. Similarly, when we analyzed UDP due to its unreliable nature its performance is poor in all the scenarios. So after analysis and result discussion this paper concludes that UDP flows perform better in the case of dense networks with little or no mobility. TCP flows perform better for high mobility scenarios

Iffat Syad et al. [18] In this research, through simulations that were carried out by using Network Simulator-2 (NS-2), the selected MANET Routing protocols i.e. DSR and DSDV were analysed in accordance with their finest performance of packets delivery rate, average end-to-end delay, and packet dropping, under TCP Vegas and TCP New Reno with mobility consideration. The simulation results indicate that DSDV has a better throughput performance but high average end-to-end delay and packet drop ratio as compared to DSR. Gayathri Janakiraman et al. [19] In the research work, MANET protocols like AODV, DSDV and DSR protocols performance analysis was investigated with TCP Reno, TCP new Reno and TCP Vegas using ns2. The analysis of TCP variants was based on the these performance metrics like Average End-to-End delay, Packet Delivery Fraction, Packet Loss, Routing Overhead and Convergence Time. These metrics were calculated by varying the node coverage area. The performance of TCP variants varies according to the routing protocols and network scenarios. Among all possibilities, the proposed TCP variant having better performance .TCP NEW RENO outperforms better as compared to other variants. DSR has performed well compared to all other protocols in terms of delivery ratio while AODV outperformed in terms of average delay. DSR generates lower overhead than AODV while DSDV generates almost constant overhead due to proactive nature. S. Mascolo et al. [20] evaluates and compares three control algorithms, 
which are Reno, Vegas and Westwood+ TCP, using the ns2 simulator, the dummy net emulator and live internet measurements. Results show that Westwood+ TCP is friendly towards Reno TCP and improves the fairness in bandwidth allocation whereas Vegas TCP is fair but it is not grab its bandwidth share when coexisting with Reno. Westwood+ improves utilization of lossy links w.r.t both Vegas and Reno and provides the highest bottleneck utilization in the presence of small capacity buffers. Md. Monzur Morshed et al. [21] In this paper, simulation results are were carried out for different TCP packets under several QoS metrics such as drop, throughput, delay and jitter. Considering the performance on the variants of TCP, Vegas show the highest efficiency and performs best. So it concludes that in terms of drop rates, delivery rates and total receiving throughput, Vegas is clearly best among the four variants.

\section{QOS BASED PERFORMANCE METRICS}

The performance metrics includes the QoS parameters such as Packet Delivery Ratio (PDR), average Throughput, average Delay, Routing Overhead and average Jitter.

Packet Delivery Ratio (PDR): PDR also known as the ratio of the data packets delivered to the destinations to those generated by the CBR sources. This metric characterizes both the completeness and correctness of the routing protocol.

$$
\text { PDR }=\frac{\sum_{1}^{n} \text { CBRrece }}{\sum_{1}^{n} \text { CBRsent }} * 100
$$

Average End to End Delay: Average End to End delay is the average time taken by a data packet to reach from source node to destination node. It is ratio of total delay to the number of packets received.

$$
A v g_{-} \text {End_to_End_Delay }=\frac{\sum_{1}^{n}(\text { CBRrecetime }- \text { CBRsentime })}{\sum_{1}^{n} \text { CBRrece }} * 100
$$

Throughput: Throughput is the ratio of total number of delivered or received data packets to the total duration of simulation time.

$$
\text { Throughput }=\frac{\sum_{1}^{n} \text { CBRrece }}{\text { simulationtime }}
$$

Normalized Protocol Overhead/ Routing Load: Routing Load is the ratio of total number of the routing packets to the total number of received data packets at destination.

$$
\text { Routing _ Load }=\frac{\sum \text { RTRPacket }}{\sum \text { CBRrece }}
$$

\section{SIMULATION RESULTS AND DISCUSSIONS}

The performance of AODV, DSDV and DSR has been analysed on TCP Variants varying number of nodes. The parameters used for simulation are summarized in Table 1.The performance metrics comprises of QoS parameters such as packet delivery ratio, average throughput, average delay, routing overhead and average Jitter.
Table 1: Simulation Parameters for Scenario

\begin{tabular}{|c|c|}
\hline Parameters & Values \\
\hline Number of nodes & $25,50,100,150,200$ \\
\hline Simulation time & 10 sec. \\
\hline Environment size & $1200 * 1200$ \\
\hline Traffic & CBR (Constant Bit-Rate) \\
\hline Queue Length & 50 \\
\hline Source node & Node 0 \\
\hline Destination node & Node 2 \\
\hline Source Type & TCP (Vegas, Westwood) \\
\hline Routing Protocols & AODV, DSDV, DSR, ZRP \\
\hline Mobility model & Random Way-Point \\
\hline Antenna type & Omni Directional \\
\hline Simulator & NS-2.34 \\
\hline Operating System & Linux Enterprise Edition-5 \\
\hline
\end{tabular}

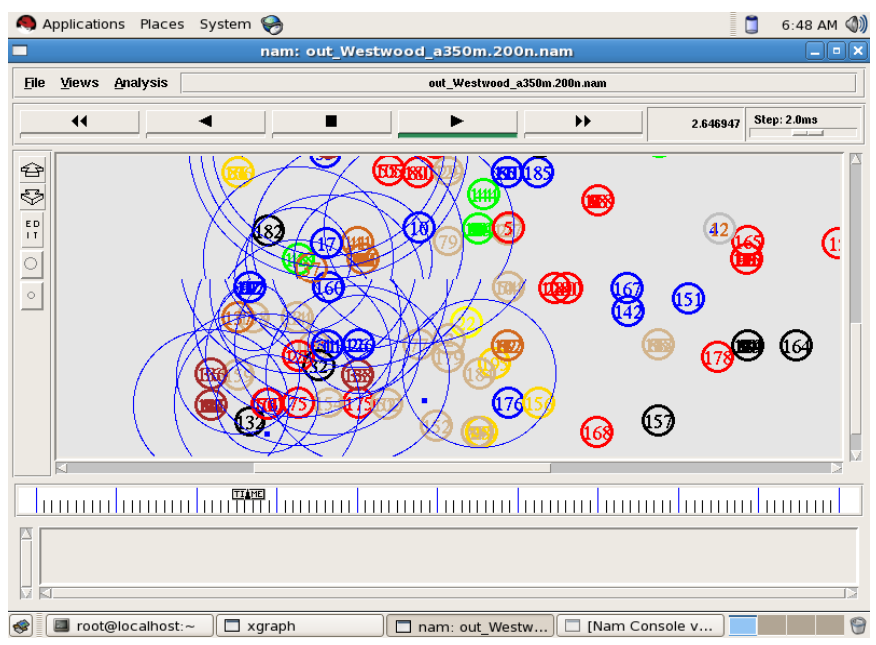

Figure 5.1 Scenario of 200 nodes of TCP westwood with AODV Routing Protocol

\section{Average Delay}

Figure 5.2 shows average delay versus number of nodes for MANET routing protocols under two TCP variants named Vegas and Westwood. It is observed that TCP Vegas having minimum average delay with AODV routing protocol followed by Westwood with AODV routing protocol whereas other TCP variant named TCP Westwood have worst performance with DSR routing protocol. TCP Vegas have better performance because it works on the estimation Round Trip Time (RTT) which detects the congestion faster and at early stages without wasting time on waiting for packet lost to conclude that congestion occurs in the network, which concludes that faster delivery of data to the destination, lower will the delay and it also improves the efficiency of the network. TCP variant Vegas gives best results with AODV routing protocol because AODV is a reactive and beacon-full routing protocol, in which convergence is fast due to the linked node communication. 


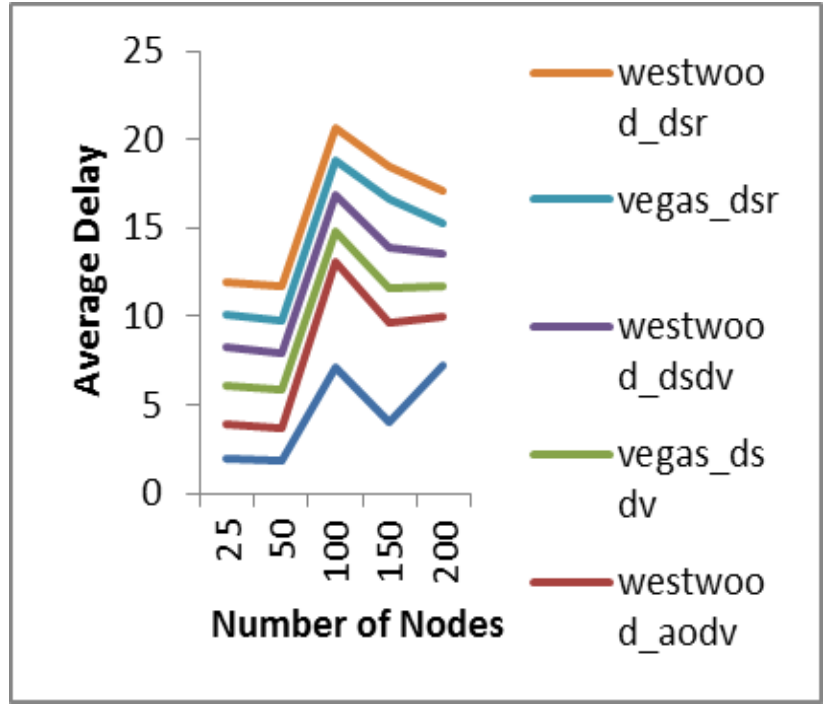

Figure 5.2: Illustrates Average Delay vs. number of node

\section{Average Throughput}

Figure 5.3 shows average throughput versus number of nodes for AODV, DSDV and DSR MANET routing protocols under two TCP variants named Vegas and Westwood. It is observed that TCP Westwood have maximum average throughput with DSR routing protocol whereas other TCP variant named TCP Vegas have worst performance with AODV routing protocol. When we increase the number of nodes in a network, more packets are dropped in the network due to the collision.

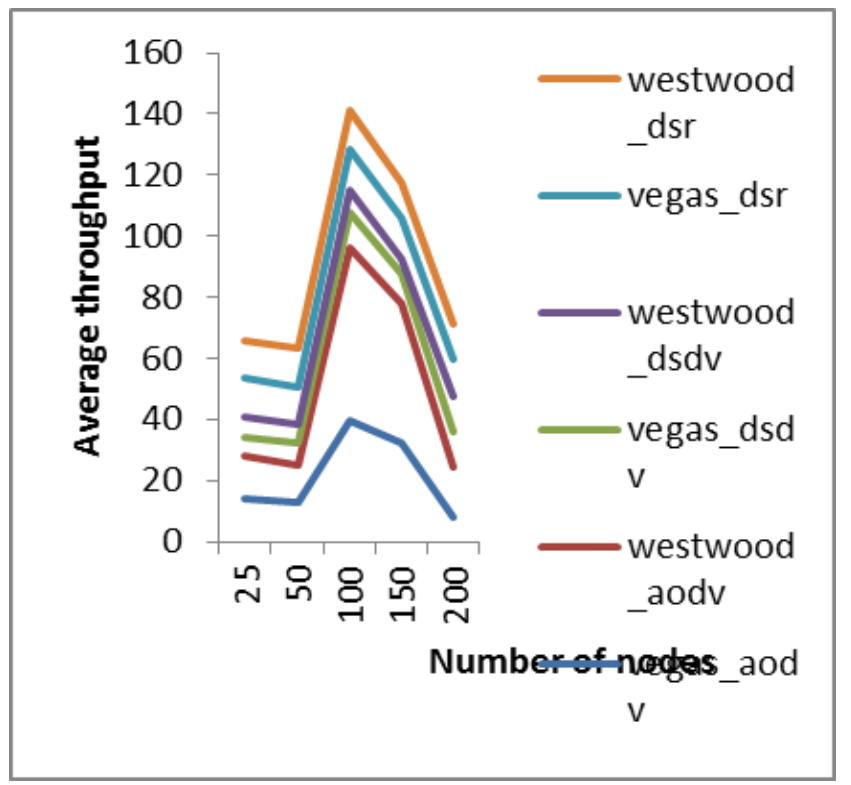

Figure 5.3: Illustrates Average throughput vs. number of node

\section{Packet Delivery Ratio}

Figure 5.4 shows Packet Delivery Ratio versus number of nodes for AODV, DSDV and DSR MANET routing protocols under two TCP variants named Vegas and Westwood. It is observed that TCP Westwood have maximum value of average throughput with DSR routing protocol whereas other TCP variant named TCP Vegas have worst performance with AODV routing protocol.

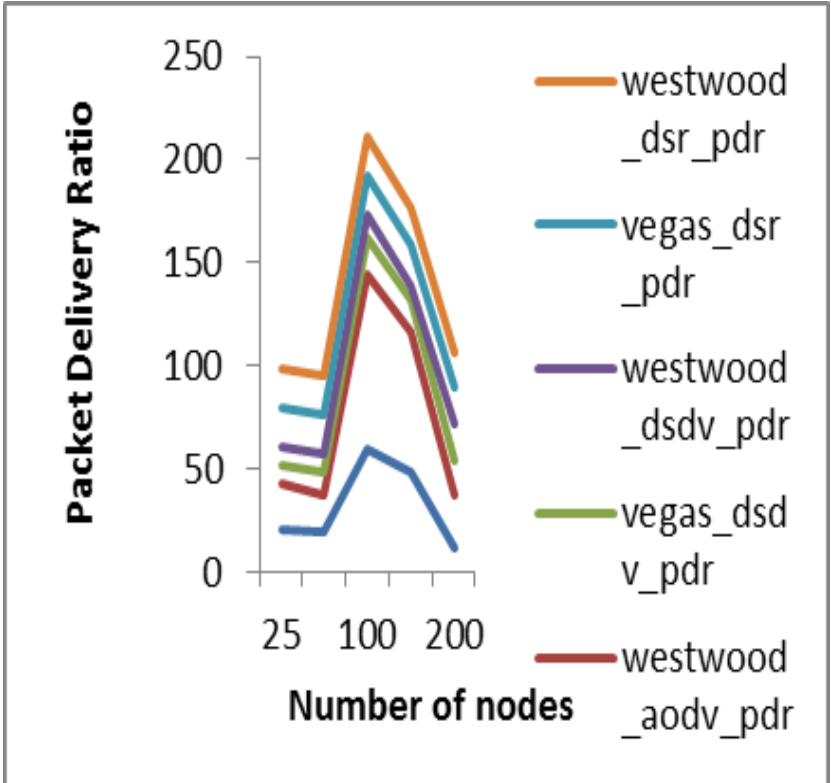

Figure 5.4: Illustrates packet delivery ratio vs. number of node

It is observed that TCP Westwood is better than other TCP variants for sending faster data to the destination and due to its faster recovery mechanism whereas TCP Vegas are giving linear delivery rate. Performance of DSR is better in PDF than AODV because in DSR route cache management is done via cache entry and only active routes are maintained in the route cache. As the number of nodes increases the neighbour density increases hence the value of PDR increases for all on demand routing protocols.

\section{Routing Overhead}

Figure 5.5 shows Routing overhead versus number of nodes for AODV, DSDV and DSR MANET routing protocols under two TCP variants named Vegas and Westwood. It is observed that TCP Vegas have minimum Routing overhead with AODV routing protocol whereas other TCP variant named TCP Westwood have worst performance with DSR routing protocol. AODV generates lower routing overhead than DSR while DSDV generates almost constant overhead due to proactive nature. AODV has lower routing overhead because it replies only once to the request arriving first and ignores the rest while DSR replies to all requests reaching a destination from a single request cycle. The major contribution to routing overhead in AODV is from RERRs, while RREPs constitute a large fraction of routing overhead in DSR. 


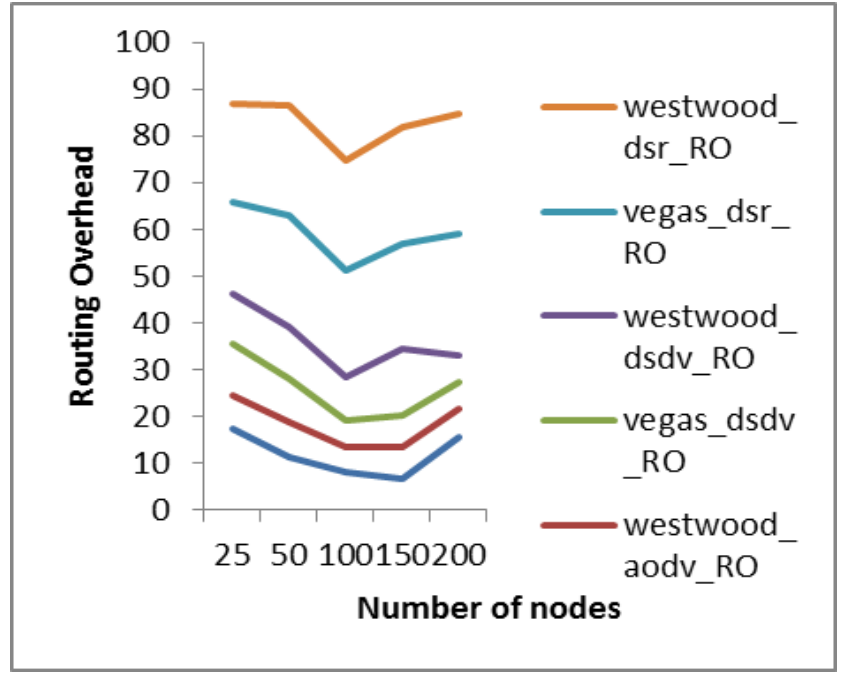

Figure 5.5: Illustrates routing overhead vs. number of node

\section{Average Jitter}

Figure 5.6 shows Average Jitter versus number of nodes for AODV, DSDV and DSR MANET routing protocols under two TCP variants named Vegas and Westwood. It is observed that TCP Vegas have minimum routing overhead with AODV routing protocol whereas other TCP variant named TCP Westwood have worst performance with AODV routing protocol. There is not much variation in the value of jitter as the number of nodes increases. TCP Vegas does not wait for loss to trigger congestion window decreases and calculate approximately the current throughput during each time, that is the main reason of Vegas having minimum Jitter.

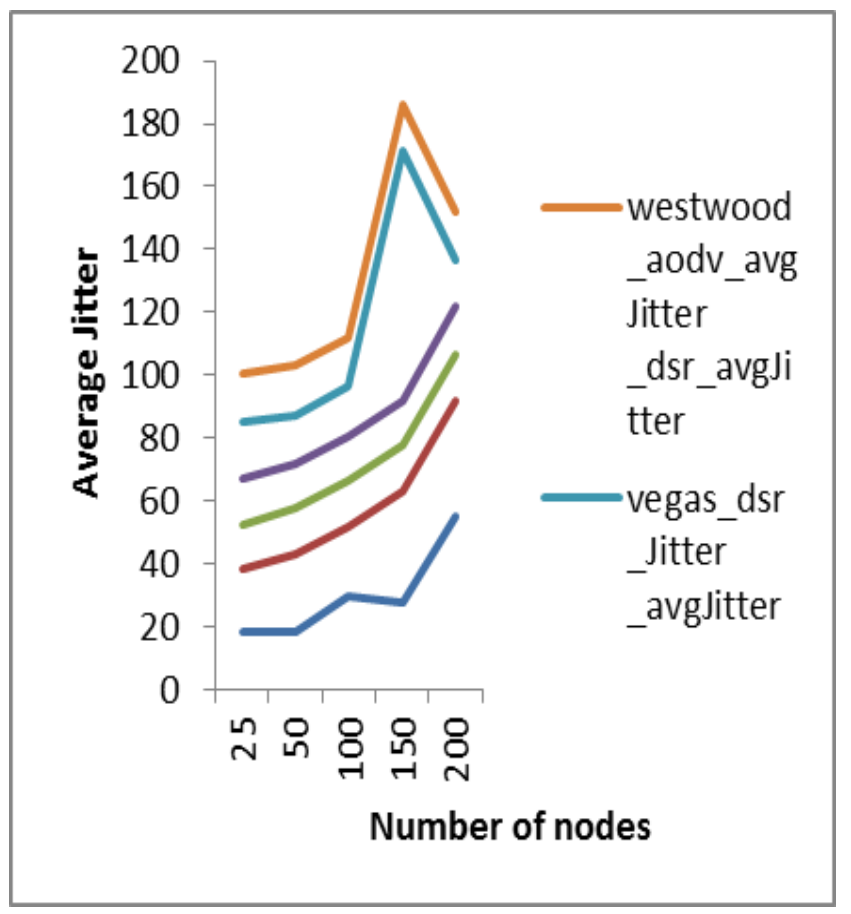

Figure 5.6: Illustrates Average Jitter vs. number of node

\section{CONCLUSION AND FUTURE WORK}

This study was conducted to simulate the performance of TCP variants over different MANET routing protocols i.e. AODV, DSDV and DSR based on average throughput, average delay, packet delivery ratio, routing overhead and average jitter. It has been found that packet delivery ratio and average throughput are better in case of TCP Westwood with DSR routing protocol with increased the number of nodes and mobility. It has been also found that average delay, routing overhead and average delay are better in case of TCP Vegas with AODV routing protocol as we increased the number of nodes and mobility.

The future work could be conducted with the analysis of MANET environment under Different quality of service (QoS) issues such as node energy consumption, issues of hidden and exposed terminals, and constraints in mobility and traffic criteria. Also more MANET protocols like Hierarchal state routing protocol (HSR), temporarily ordered routing protocol (TORA), Pre-emptive Ad-hoc on-demand distanceVector routing protocol (PAODV). Dynamic MANET OnDemand (DYMO) can be added for better analysis and comparison of protocols under different TCP variants. Other TCP variants can also be tested. In future more parameters will be taken into consideration such as different traffic scenarios, congestion window size, number of connections etc.

Simulation Based Analysis of TCP Variants over MANET Routing Protocol.

\section{REFERENCES}

[1] Kapang Lego, Pranav Kumar Singh, Dipankar Sutradhar, "Comparative Study of Ad-hoc Routing Protocol AODV, DSR and DSDV in Mobile Ad-hoc NETwork", Indian Journal of Computer Science and Engineering Vol. 1(4) , 2011.

[2] S.R. Birdar, Hiren H D Sarma, Kalpana Sharma, Subir Kumar Sarkar , Puttamadappa C, Performance Comparison of Reactive Routing Protocols of MANETs using Group Mobility Model”, International Conference on Signal Processing Systems, 2009.

[3] R.Ramanathan, J.Redi, and B.Technologies, "A Brief Overview of Ad-hoc Networks: Challenges and Directions", IEEE Communications Magazine, Vol. 40(5), pp. 20-22, 2002.

[4] Krishna Gorantala, "Routing Protocols in Mobile Ad-hoc Networks", Umea University, Sweden, Vol. 38(5), 2012.

[5] C.E Perkins, E.M. Royer, and S. Das, -Ad-hoc Ondemand Distance Vector(AODV), RFC 4561, 2005.

[6] C.E. Perkins and E.M. Royer, -Ad-hoc On-Demand Distance Vector RoutingProceedings of the 2nd IEEE Workshop on Mobile Computing Systems and Applications, New Orleans, LA, pp. 90-100, 1999.

[7] Abdul Hadi Abd Rahman and Zuriati Ahmad Zukarnain,"Performance Comparison of AODV, DSDV and I-DSDV Routing Protocols in MANETs" European Journal of Scientific Research, ISSN 1450216X Vol.41(4), pp. 566-576, 2009.

[8] C.Perkins, E.Royer and S Das, -Ad-hoc on demand distance vector (aodv) routing, $\|$ in IETF Internet Draft(work in progress), 2000. 
[9] D. B. Johnson and D. A. Maltz, -Dynamic Source Routing in Ad-hoc Wireless Networks, Mobile Computing, Chapter 5, pp. 154-181, Kluwer Academic Publishers, 1996

[10] J. Postel, -Transmission Control Protocol, RFC 794, 1981.

[11] M. Mathis, J. Mahdavi, S. Floyd, A. Roma, RFC 2018: TCP Selective acknowledgment options, 1996.

[12] N Vetrivelan, A V Reddy - Performance Analysis of Three Routing Protocols for Varying MANET Size Proceedings of the International Multi-Conference of Engineers and Computer Scientists 2008 Vol. 2, pp. 1921,2008

[13] Yuvaraju B N, Dr. Niranjan N Chiplunkar "Scenario Based Performance Analysis of Variants of TCP Using NS2-Simulator" International Journal of Computer Aplications, Vol. 4(9), 2010.

[14] B. S. Yew, B. L. Ong and R. B. Ahmad "Performance Evaluation of TCP Vegas versus Different TCP variants in Homogeneous and Heterogeneous Networks by Using Network Simulator 2" International Journal of Electrical and Computer Sciences IJECS- IJENS Vol. 11( 04) .

[15] Macura, a Missoni, E \& kordic, z "comparison of westwood, new reno and vegas tcp congestion control" international daaam symposium, vol. 24(1).

[16] Rajneesh Kumar Gujral, Manpreet Singh, Jyoti Kalra, "Impact of Scalability with respect to Mobility and Zone Size on Zone Routing Protocol over MANETs" International Conference on Computing Sciences, December 2012, pp. 343-348.

[17] Rajneesh Kumar, Sandhya Umrao "Performance based Reliable Data Communication Analysis on TCP, UDP by varying nodes, mobility speed and zone radius over ZRP” International Journal of Engineering Research and Applications (IJERA), pp. 34-39, March 2014.

[18] Iffat Syad, Sehrish Abrejo and Asma Ansari "analysis of proactive and reactive manet routing protocols under selected tcp variants" International Journal of Ad-hoc, Sensor \& Ubiquitous Computing (IJASUC) Vol. 4(4), 2014.
[19] Gayathri Janakiraman, T. Nirmal Raj, R.M. Suresh "AODV, DSDV, DSR Performance Analysis with TCP Reno, TCP New Reno, TCP Vegas on MANETs using NS2" International Journal of Computer Applications (0975 - 8887) Vol.19, 2014.

[20] S. Mascolo, A. Grieco, G. Pau, C. Casetti, "End-to-End Bandwidth Estimation toImprove Wireless Link Utilization", Vol. 23(2):235-248, 2005

[21] Md. Monzur, Morshed, Mehtah Ur Rahman and Md. Rafiqul Islam, "An Empirical Study on variants of TCP over AODV routing protocol in MANET", Vol. 6(16), 2012.

\section{AUTHOR'S PROFILE}

Navreet Kaur is a M.Tech Student in Department of Computer Science and Engineering in the M.M. Engineering College, M.M. University Mullana, Ambala. She obtained her M.Tech (Computer Science and Engineering) from M.M. University Mullana, Ambala and B.Tech (IT) from PTU, Jalandhar.

Sandhya Umrao working as a Assistant Professor (IT) in Galgotia College of Engineering and Technology Greater Noida (U.P.) since July 2007. She has received her M.Tech. (Computer Science and Engineering) From Kurukshestra University Kurukshetra (Haryana) in 2007. She supervised 2 M. Tech students. Currently she is pursuing $\mathrm{PhD}$ from M,M,University Mullana Ambala in the area of Mobile Ad hoc Network. Her research area includes Wireless Sensor Networks, Reliability Theory, Artificial Intelligent and Cryptography.

Dr. Rajneesh Kumar Gujral is working as Professor in the Department of Computer Science and Engineering, M.M Engineering College, M. M. University Mullana, Ambala. He obtained his $\mathrm{PhD}$ (Computer Science and Engineering) in 2012 under faculty of engineering, M.M University, Mullana, MTECH (IT) in 2007 from University School of Information Technology, GGSIP University Delhi and BE (Computer Science and Engineering) in 1999 from Punjab Technical University (PTU), Jalandhar (Punjab). He supervised $28 \mathrm{M}$. Tech, $1 \mathrm{M}$. Phil and currently supervising $8 \mathrm{PhD}$ research scholars. He has about 40 publications in International Journals and Conferences. His research area includes Cloud Computing, Wireless Communications, Mobile Ad hoc \& Sensor based Networks and Network Security. 